\title{
EINE SPÄTANTIKE ÄRMELBORTE DER SAMMLUNG TAMERIT (T 34) IN DER PAPYRUSSAMMLUNG DER ÖSTERREICHISCHEN NATIONALBIBLIOTHEK ${ }^{1}$
}

\author{
Einleitung
}

Der spätantike Stoff mit der Inventarnummer T 34 (Abb. 1) ist Teil einer deutschen Privatsammlung genannt „Tamerit" ${ }^{\text {“2 }}$. Große Teile der Sammlung und somit auch das Stofffragment T 34 sind derzeit als Leihgabe in der Papyrussammlung der Österreichischen Nationalbibliothek (ÖNB) aufbewahrt, wo sie in mühevoller Kleinarbeit konserviert und wissenschaftlich bearbeitet werden.

Das Herkunftsland dieses spätantik-frühislamischen Textils (T 34), das wahrscheinlich von sog. koptischen ${ }^{3}$ Webern geschaffen wurde, ist Ägypten ${ }^{4}$. Die Gesamtbreite des Stoffes beträgt an der rechten Kante 20,5 cm und an der linken $21,2 \mathrm{~cm}$; die Gesamtlänge $33 \mathrm{~cm}$. Die eingewirkte ${ }^{5}$ Borte $(12,5 \times 26,5 \mathrm{~cm})$, die sich als Einsatzstück auf einer Ärmeltunika ${ }^{6}$ als Ärmelborte befand, zeigt ein zentrales Medaillon, das von vier rechteckigen Feldern flankiert wird. Die polychromen Figuren und Ornamente sind in der Technik der Buntwirkerei gearbeitet. Diese Art der Verzierung kann man für die sog. koptischen Stoffe als typisch ansehen.

\section{Beschreibung}

Die eingehende Betrachtung der Ärmelborte T 34 beginnt mit dem zentralen Medaillon, um dann mit dem linken, oberen Figurenfeld fortzufahren.

Das Medaillon ( $6 \times 7 \mathrm{~cm}$; Abb. 2) sowie die vier rechteckigen Figurenfelder sind auf einen purpurnen Hintergrund gesetzt. Eingerahmt wird es an der linken und rechten Seite von je zwei Rechtecken; oben und unten von Borten mit polychromen, maritimen Motiven. Diese besonderen Dekorationen heben das Medaillon und somit die Mitte der Ärmelborte hervor. In den vier,

\footnotetext{
${ }^{1}$ Die Ärmelborte T 34 ist in meiner Diplomarbeit Ch. Pflegerl, Eine koptische Ärmelborte der Sammlung Tamerit (T 34) in der Papyrussammlung der Österreichischen Nationalbibliothek. Bearbeitet anhand der Textiltechnik und spätantiken Vergleichsbeispiele. Unpubl. Dipl. Wien 2002, ausführlich behandelt. Ich danke HR Prof. Dr. Hermann HaRRAUER für die Publikationserlaubnis.

${ }^{2}$ Da der Sammler nicht sehr viel über seine „Schätze“ preisgibt, war es mir unmöglich, Näheres über Fundort und Erwerb des Stoffes T 34 herauszufinden.

${ }^{3}$ Die Bezeichnung „koptisch“ wurde der Bevölkerung Ägyptens von den Arabern bei deren Eroberung 640-642 n. Chr. gegeben, die den griechischen Namen Aỉúrtıऽ in „kibt“ umwandelten.

${ }^{4} \mathrm{Da}$ dieses Stück durch einen Privatsammler angekauft wurde, fehlen jegliche Informationen, was den Fundzusammenhang betrifft.

${ }^{5} \mathrm{Zu}$ „Wirkerei“ siehe A. SeILER-BALdINGER, Systematik der textilen Techniken (Basler Beiträge zur Ethnologie 32). Basel 1991, 72.

${ }^{6}$ Die ägyptische Ärmeltunika, auch kreuzförmige Tunika genannt, erhielt ihre spezielle Form am Webstuhl selbst. Sie wurde nach einem exakten, vorher vereinbarten Plan gewoben. Sie ist gewöhnlich reich mit gewirkten Ornamentbändern oder mit Ornamenten aus Wolle, die durch ihre Farbe bestechen, verziert. Die Breite des Stoffes formt die Länge des fertigen Kleidungsstückes, was bedeutet, dass die Schussfäden längs liegen und die Kettfäden quer darüber. M. HALD, Ancient textile techniques in Egypt and Scandinavia. A comparative study. AA 17 (1946) 68.
} 


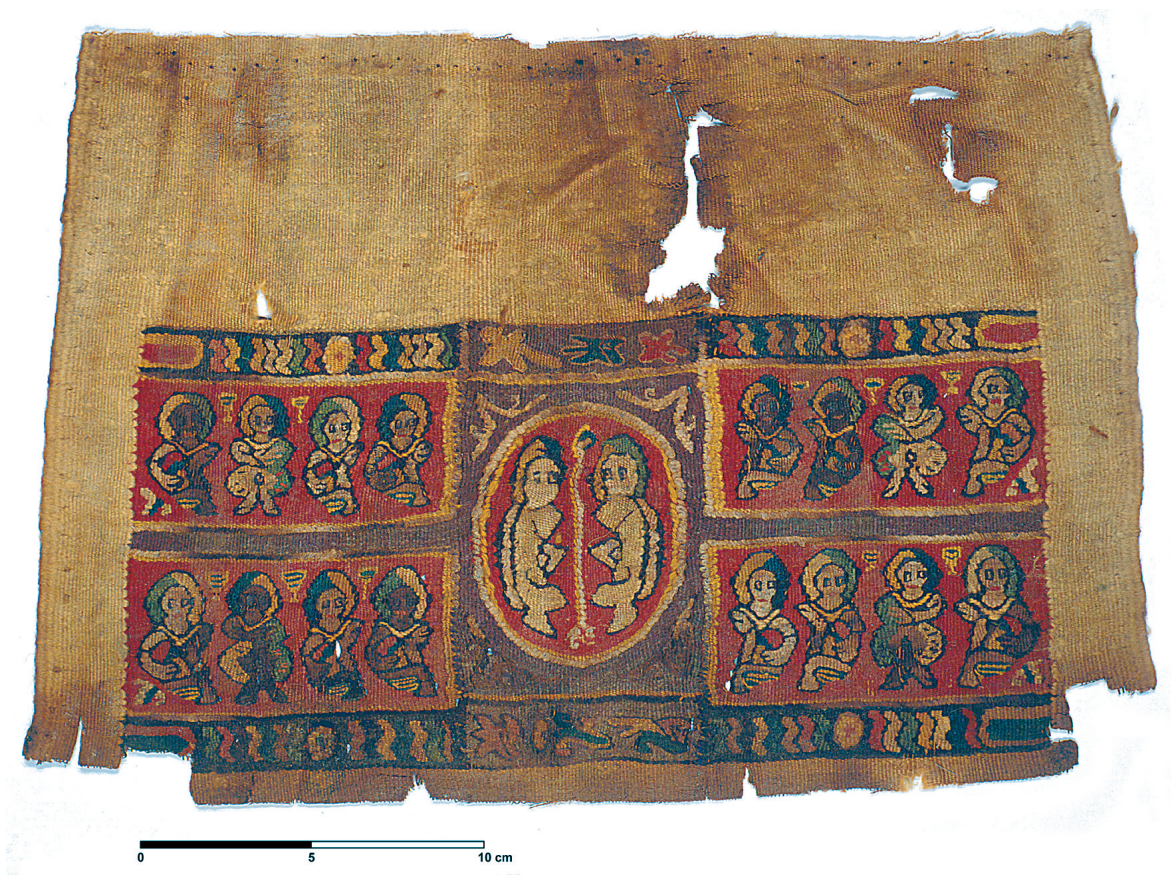

Abb. 1: Ärmelborte T 34, $33 \times 21,5$ cm (Foto: I. OENTRICH, ÖNB)

das Medaillon umgebenden Ecken, sind stilisierte pflanzliche Darstellungen zu sehen. Das Medaillon selbst ist von einer weißen und einer gelben Umrisslinie eingefasst. Darin eingeschrieben sind zwei Figuren, die sich mit ihrem Körper im Profil dem Gebilde in deren Mitte zuwenden. Ihre Köpfe und da besonders ihre großen Augen sind frontal auf den Betrachter gerichtet. Auf dem Kopf tragen sie eine nimbusartige „Haube“, die zweifarbig ist und ihr Haar vollständig verdeckt. Bewegung und Form ihrer Arme und Hände sind nicht sicher zu klären. Es scheint, als ob sie den jeweils zum Betrachter gerichteten Arm auf ihre andere Schulter gelegt hätten. Das Gebilde auf ihrem Rücken könnte man sowohl als überproportionierten Arm als auch vielleicht als Flügel oder Gewand ansprechen. Ein blattartiges Motiv ist über dem Bauch, der mittels braunen Punkt als Nabel angedeutet ist, dargestellt. Ihre Beine scheinen angewinkelt zu sein. Die Füße wirken klobig ohne Angabe der Zehen. Die Figuren passen sich in ihrer Form dem Medaillon an und sind mit braunen Umrisslinien versehen.

Das schnurartige Gebilde, das die heraldische Erscheinung der Figuren bewirkt und diese voneinander trennt, besteht aus zwei Bändern (gelb und weiß), die mit einem flaschengrünen Rund beginnen und ein wurzelartig aufgezwirbeltes Ende besitzen. Die Hintergrundfarbe des Medaillons ist ziegelrot.

Die das Medaillon flankierenden Rechtecke sind in ihrer Motivik und Darstellungsweise fast identisch. Sie werden ebenfalls von weißen und gelben Umrisslinien eingerahmt. Der Hintergrund ist ziegelrot (siehe Medaillon). Die Personen bewegen sich zur Mitte hin, wobei ihre Körper im Profil und ihre Köpfe en face gezeigt werden. Die jeweils dritte Figur von der Mitte aus gesehen sticht durch ihre andersartige Darstellung hervor: Sie steht frontal zum Betrachter und scheint in ihrer Bewegung starrer als die anderen Figuren. In den äußeren, unteren Ecken sind vegetabile Motive zu sehen. Zwischen den Köpfen der Figuren befinden sich einmal drei, dann zwei kelchartige Gebilde. Unterschiedlich sind die Hautfarben der Figuren.

Da die vier Figurenfelder einander sehr ähneln, wird beispielhaft das linke, obere Rechteck $(4,2 \times 9,5 \mathrm{~cm} ;$ Abb. 3$)$ beschrieben, um im Anschluss die Unterschiede zu den anderen Feldern hervorzuheben.

Links außen beginnt das Feld mit einer dunkelfarbigen Figur, die man auf Grund der gezeigten Bewegung als Tänzer ansprechen könnte. Der frontal ausgerichtete Kopf trägt deut- 


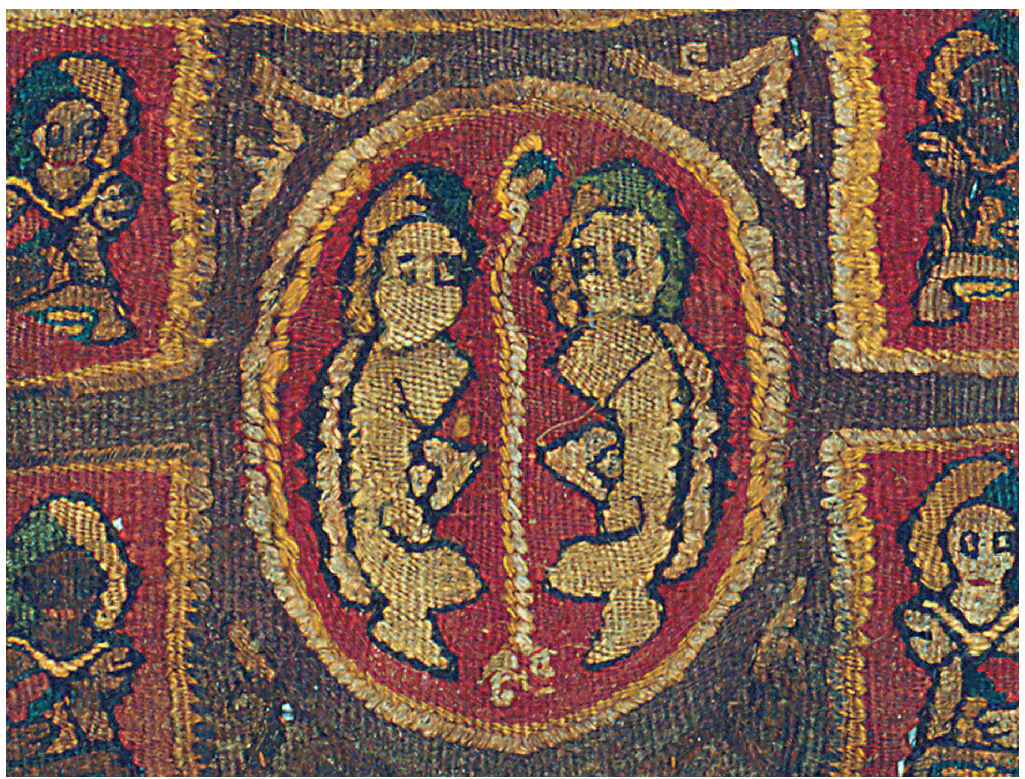

Abb. 2: Ärmelborte T 34, Medaillon, $6 \times 7$ cm (Foto: I. Oentrich, ÖNB)

lich zu erkennende braune Augen und einen roten Mund, wobei die großen Augen hervorstechen. Den Kopf umgibt wiederum eine zweifarbige, nimbusartige „Haube“. Um den Hals ist eine amulettartige Schnur gelegt, die auf eine Kette oder einen Umhang deuten könnte.

Der Körper des „Tänzers“ ist im Profil dargestellt. Er streckt den linken, stark verkürzten Arm nach vorne, wobei die Finger durch braune Striche angedeutet sind. Sein rechter, überproportionierter Arm umfängt ein rundes, mehrfarbig gestreiftes Gebilde. Es ist nur ein Bein zu sehen, das eine Art Tanz- oder Schrittbewegung zur Mitte hin vollführt. Die untere Seite des Beines besitzt eine grün-gelbe Stelle, was auf eine Hose oder Ähnliches hinweisen könnte. Die Zehen sind nicht dargestellt.

Die Figur daneben besitzt denselben en face dargestellten Kopf mit zweifarbiger, nimbusartiger „Haube“ und große Augen. Ganz anders hingegen ist die Wiedergabe des Körpers, die hellere Hautfarbe und die Hervorhebung der Figur durch zwei kelchartige Gebilde. Die Person ist frontal ausgerichtet. Beide Arme scheinen ineinander verschränkt zu sein, was ein unbewegliches Bild ergibt. Die Beine haben an ihren Außenseiten einen gelben und grünen Streifen, der mit strichartigen Verzierungen dekoriert ist. Dazwischen ist ein runder, brauner Kreis zu sehen. Die Füße sind nach außen gespreizt und tragen einen braunen Strich, vielleicht als Andeutung der Zehen. Ein amulettartiges Band ist ebenfalls (siehe oben) um den Hals geschlungen.

Es folgt ein Tänzer mit heller Hautfarbe und einer mit dunkler, die, wie schon erwähnt, gleich dargestellt sind.

Das linke, untere Figurenfeld $(4,5 \times 9,5 \mathrm{~cm})$ ist bis auf die Hautfarben der Figuren und drei kelchartige Gebilde mit den oben beschriebenen Rechtecken ident. Das gleiche gilt für die beiden rechten Figurenfelder (Abb. 4).

Die vier Rechtecke werden von Zierborten mit geometrischen Motiven abgeschlossen. Diese Borten sind schmäler als jene des Medaillons und beginnen außen mit einem Zungenblattmotiv, gefolgt von einer s-förmigen Kette, in deren Mitte sich ein Kreis mit kreuzartigem Dekor befindet.

Der Aufbau der Ärmelborte lässt also eine Dreiteilung erkennen: 1. die linken Figurenfelder mit den abschließenden, schmäleren Zierborten, 2. der hervorgehobene Mittelteil mit Medaillon und breiteren, maritimen Borten und 3. die rechten Figurenfelder, die spiegelbildlich die linken wiederholen.

Der Erhaltungszustand des Stoffes ist gut. Es sind mehrere Fehl- und Rissstellen (vielleicht auch Schnittstellen) sowie Verbräunungen im Grundgewebe zu erkennen. Die Ärmelborte zeigt 
sehr kräftige und sich voneinander absetzende Farben („Purpur“, Hellgrün, Flaschengrün, Ziegelrot, Weiß, Gelb, Hellgelb, Kaminrot und verschiedenste Brauntöne), die durch äußere Einflüsse und Abnützungserscheinungen gelitten haben und daher oft eine verfälschte Farbigkeit zeigen? .

\section{Technik $^{8}$}

Da die Kenntnis der Webtechnik eines Zierstückes zum besseren Verständnis desselben beiträgt und oft auch bei der Interpretation und Datierung hilfreich sein kann, sollte sie bei einer umfassenden, wissenschaftlichen Bearbeitung eines Stückes nicht außer Acht gelassen werden.

7 Zur Farbigkeit des Objekts siehe Ch. Pflegerl, Eine koptische Ärmelborte der Sammlung Tamerit ( $T$ 34) in der Papyrussammlung der Österreichischen Nationalbibliothek. Bearbeitet anhand der Textiltechnik und spätantiken Vergleichsbeispiele, 25f.

${ }^{8}$ Weiterführende Literatur zur Textil- und Webtechnik: D. AHRENS (Hg.), Trierer Wohn- und Tischkultur. Katalog des Städtischen Museums Simeonstift Trier. Textilien 3. Trier 1987; A. BAGINSKI - A. TIDHAR, Textiles from Egypt $4^{\text {th }}-13^{\text {th }}$ Centuries C. E. [Catalogue of the Exhibition at the L. A. Mayer Memorial Institute of Islamic Art]. Jerusalem 1980; D. L. CARROLL, Looms and Textiles of the Copts. First Millennium Egyptian Textiles in the Carl Austin Rietz Collection of the California Academy of Sciences (Memoir 11). Washington - Seattle - London 1988; J. FrICKE, Textiles Gestalten. Weben, Knüpfen, Batiken, Sticken. Objekte und Strukturen. Niedernhausen 1982; M. GÄCHTER-WeBER, Koptische Gewebe. Katalog, Industrie- und Gewerbemuseum des Kaufmännischen Directoriums St. Gallen. St. Gallen 1981; M. HALD, Ancient textile techniques in Egypt and Scandinavia. A comparative study. AA 17 (1946) 49-98; J. LAMMER, Freies Weben. Wege und Möglichkeiten phantasievoller Textilgestaltung auf kleinen Rahmen. Ravensburg 1982; A. Pekridou-Gorecki, Mode im antiken Griechenland. Textile Fertigung und Kleidung (Beck's archäologische Bibliothek). München 1989; F. RABENSTEIN - K. STICHLER - L. VLASAKHeINZ, Unterrichtssequenzen Werken, Textiles Gestalten: konstruktives Gestalten im integrativen Unterricht der Hauptschule. Donauwörth 1999;

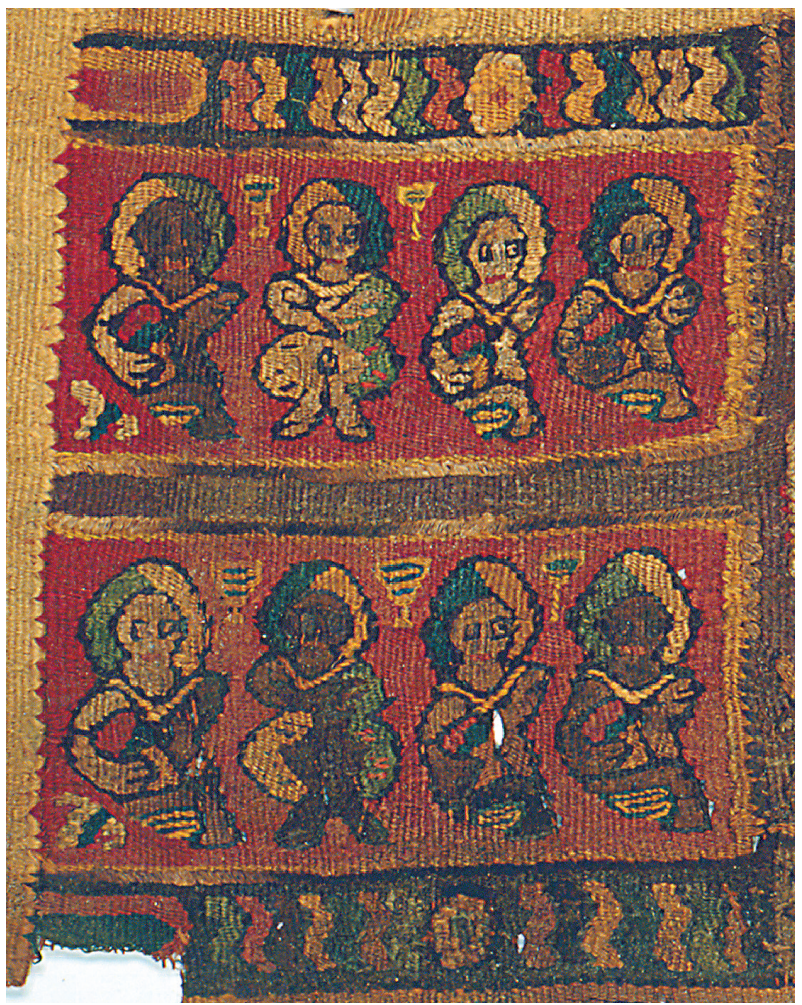

Abb. 3: Ärmelborte T 34, die zwei linken, rechteckigen Figurenfelder, je 4,5 × 9,5 cm (Foto: I. OentRICH, ÖNB)

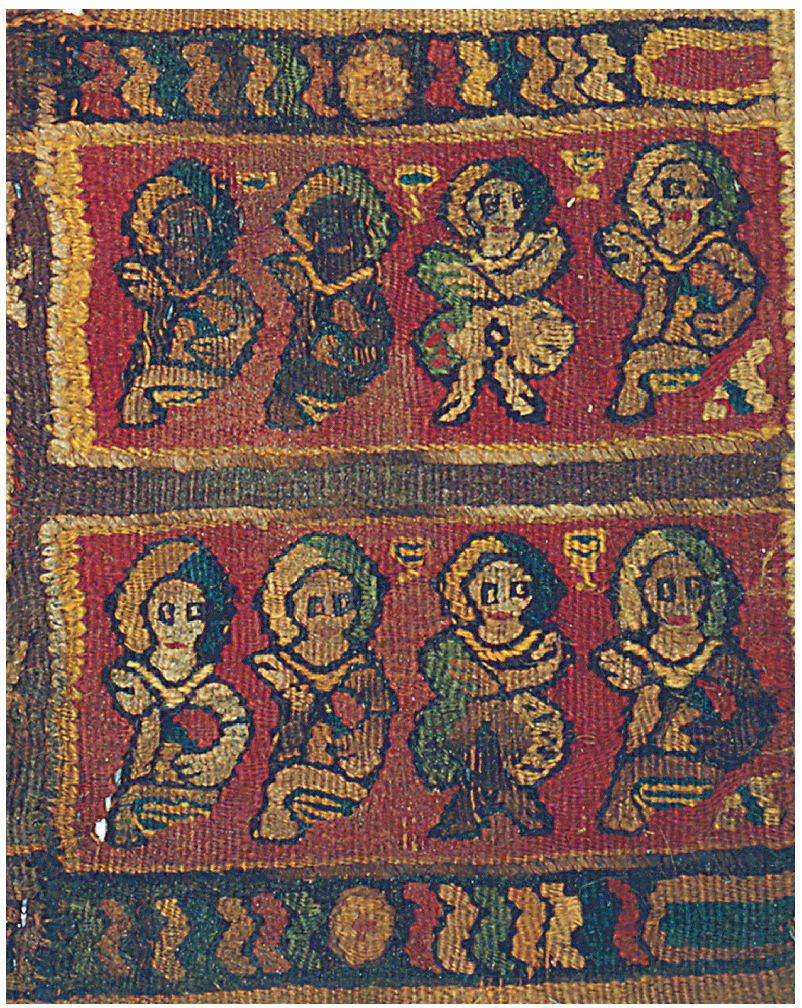

Abb. 4: Ärmelborte T 34, die zwei rechten, rechteckigen Figurenfelder, je 4,5 × $10 \mathrm{~cm}$ (Foto: I. OENTRICH, ÖNB) 
Die Ärmelborte T $34^{9}$ umgibt ein helles, wollenes Grundgewebe, das in Leinwandbindung ${ }^{10}$ ausgeführt wurde. Die verstärkten Webkanten, an der linken und rechten Seite in Kettrichtung gelegen, sind sehr gut zu erkennen. An der oberen Kante sind Nählöcher zu sehen, die vielleicht von einer Zweitverwendung oder einer Konservierungstätigkeit stammen. Die Fadendichte des Grundgewebes, die Aufschluss über die Feinheit gibt, beträgt 22 Kettfäden $\times 45$ Schussfäden pro $\mathrm{cm}^{2}$.

Die Ärmelborte besteht ebenfalls aus Wolle und wurde in das umgebende Grundgewebe eingewirkt. Die Wirkerei ist ein spezielles Verfahren, bei dem der Schuss leinwandbindig eingetragen wird, jedoch nur soweit führt, wie das Muster es verlangt. Es entstehen Schlitze zwischen den einzelnen Farb- und Musterflächen ${ }^{11}$. Diese Schlitze werden durch die sog. verzahnte Wirkerei miteinander verhängt, was eine eigene Musterbildung hervorruft, die besonders an den Außenkanten der Ärmelborte zu erkennen ist. Die Fadendichte der Ärmelborte beträgt 24 Kettfäden $\times 58$ Schussfäden pro $\mathrm{cm}^{2}$ und ist somit feiner ausgeführt als das Grundgewebe, was allgemein meistens der Fall ist.

Weitere Besonderheiten der Ärmelborte T 34 sind die Verwendung der Sumakhschlinge und der „Fliegenden Nadel“, wobei es sich um eine Webtechnik handelt.

Bei letzterer werden Schussfäden ohne Rücksicht auf die Kettfäden in einer Art Sticktechnik über das Gewebe gelegt. So kann man Details wie Augen, Mund und die Angabe der Finger als Binnenmalerei „zeichnen“.

Die Sumakhschlinge ist eine Stichart, bei der Schlingen erhaben über zwei Kettfäden geführt werden. Dadurch entstehen Musterlinien, die Figuren und Ornamente der Ärmelborte T 34 braun einrahmen.

Die Betrachtung der Rückseite sollte man nie außer Acht lassen, da oft sehr überraschende Erscheinungen zu Tage treten, wie bei T 34, wo ein Nähfaden zu finden war, der auf der Vorderseite nicht sichtbar ist.

Abschließend kann man sagen, dass die Ärmelborte T 34 eine sehr regelmäßige und sorgfältige Handwerksarbeit ist, die allen technischen Anforderungen der Buntwirkerei genügt.

\section{Deutung}

Im Folgenden soll ein Versuch unternommen werden die einzelnen Figuren und Figurengruppen zu interpretieren.

Zuerst wird der Aufbau der Ärmelborte T 34 betrachtet. Es kann anhand von Vergleichsbeispielen festgestellt werden, dass diese Art der Dreiteilung mit zentralem Medaillon und umgebenden Ornamentfeldern eine durchaus übliche gewesen ist. Wobei gesagt werden muss, dass die Ärmelborte so angebracht war, dass die Figuren und Ornamente zum Betrachter gerich-

\footnotetext{
A. SeILer-Baldinger, Systematik der textilen Techniken (Basler Beiträge zur Ethnologie 32). Basel 1991; A. STAUfFER - M. Hill - H. C. Evans - D. WALKer, Textiles of Late Antiquity [Catalogue of the Exhibition at The Metropolitan Museum of Art from December 14, 1995 to April 7, 1996]. New York 1995; Th. K. Thomas, Carnegie Museum of Natural History. Textiles from Medieval Egypt, A. D. 300-1300. Pittsburgh 1990; B. TiETZEL, Geschichte der Webkunst. Technische Grundlagen und künstlerische Traditionen (DuMont-Monumente). Köln 1988; E. VITI, Kleine Textil- und Stoffkunde. Wien 1981.

${ }_{9}^{9}$ Zur genaueren Abhandlung der Webtechnik von T 34 siehe Ch. PfLEgerl, Eine koptische Ärmelborte der Sammlung Tamerit, 14-24.

${ }^{10}$ Leinwandbindung ist die einfachste Verkreuzung von längsgerichteten Fäden (Kettfäden) und den quergerichteten Fäden (Schussfäden). Siehe E. ViTI, Kleine Textil- und Stoffkunde, 1 und J. Fricke, Textiles Gestalten. Weben, Knüpfen, Batiken, Sticken. Objekte und Strukturen, 7.

${ }^{11}$ Sie wird auch Schlitz- oder Buntwirkerei genannt. Siehe A. SeILER-BALdinger, Systematik der textilen Techniken, 72f.; Th. K. Thomas, Carnegie Museum of Natural History. Textiles from Medieval Egypt, A. D. 300-1300, 61; B. TIETzeL, Geschichte der Webkunst. Technische Grundlagen und künstlerische Traditionen, 254 und A. BAgInsKi - A. Tidhar, Textiles from Egypt $4^{\text {th }}-13^{\text {th }}$ Centuries C. E., Glossary 33.
} 


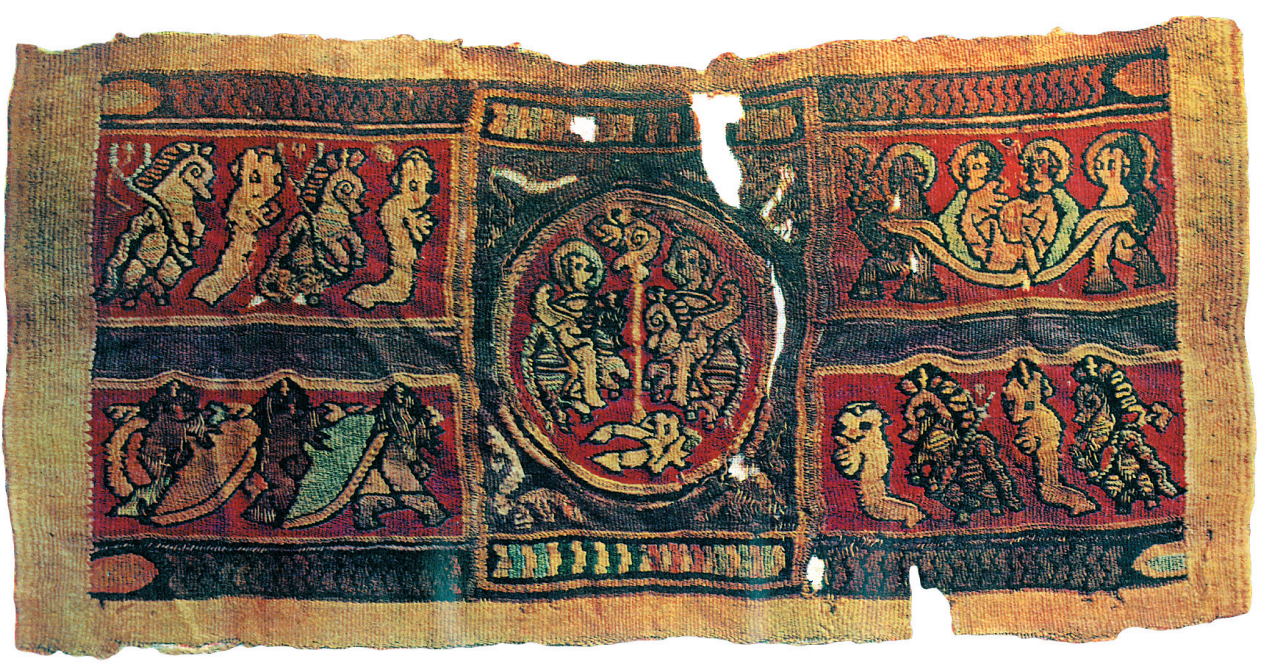

Abb. 5: Ärmelborte mit Mensch- und Tierdesign, Kanegafuchi Sammlung (Foto nach K. AkAshi [Hg.], Coptic Textiles [wie Anm. 12], Abb. 65)

tet waren und natürlicherweise das Medaillon als Mittelstück auf dem Arm auflag, also die wahrscheinlich wichtigere, teilweise verschlüsselte Botschaft beinhaltete.

Ein Stofffragment mit exakt dem gleichen Aufbau ist in der Kanegafuchi Sammlung ${ }^{12}$ zu fin- $^{-}$ den. Diese Ärmelborte mit Mensch- und Tierdesign, wahrscheinlich aus dem 6./7. Jh. (Abb. 5), trägt auch dieselbe Farbigkeit wie T 34. Sehr ähnlich sind die Figuren mit der nimbusartigen Kopfbedeckung und das Mittelmedaillon mit trennendem Gebilde, das von vegetativen Eckornamenten umgeben ist. Unterschiedlich ist die Abbildung von tierartigen Wesen, Bewegung und Handlungen der Figuren.

Weiters ist jeweils eine Figur in den Rechtecken durch ihre frontale Haltung und durch ihre Positionierung zwischen zwei kelchartigen Gebilden auffallend. Hier war ein Stoff (Abb. 6) ${ }^{13}$, der den trunkenen Dionysos zeigt, welcher von Pan gestützt wird, bei dem Versuch einer Deutung sehr hilfreich. Er befindet sich in Boston im Museum of Fine Arts und wird in das 5.-7. Jh. datiert. Zum einen sind beide Gestalten nimbiert, was bei mythologischen Figuren nichts Besonderes ist, zum anderen ist das Ziegenfell des Pan sehr gut zu erkennen. Es ist mit Haarbüscheln, die durch mehrere Striche gebildet werden, dargestellt. Das führt beim Textil T 34 zu den strichartigen Verzierungen der Beine der frontalen Gestalt, die man ebenfalls als Bocksbeine sehen könnte. Weiters zu vergleichen sind die Hufe des Pan, die wieder durch einen Strich angegeben sind, was bei T 34 ebenfalls der Fall ist. Im dionysischen Bereich bleibend kann weiterführend das runde Gebilde zwischen den Beinen als Geschlecht angesehen werden. Diese Art der Darstellung wäre demzufolge typisch für Pan, nämlich Beine mit Fellen und Hufe und das Zeigen des Geschlechts.

Wenn die frontale Gestalt in den Figurenreihen als Pan anzusprechen ist, was könnten oder müssten die zwölf Figuren beidseitig der vier frontalen Gestalten darstellen?

Eine Clavusdekoration (Abb. 7) aus der C. A. Rietz Sammlung, 9. oder 10. Jh., enthält Rechtecke mit Gruppen von Tänzern im Wechsel mit Rechtecken, auf welchen ein kunstvoller, symmetrischer Baum zu sehen is ${ }^{14}$. Hier werden die Figuren als Tänzer angesprochen. Sie

${ }^{12}$ K. AKashi (Hg.), Coptic Textiles Vol. 1-16 from burying grounds in Egypt. Kanegafuchi Spinning Company Collection. Kyoto 1992, Abb. 65, siehe auch ebd., Abb. 36.

${ }^{13}$ M.-H. Rutschowscaya, Coptic Fabrics. Paris 1990, 18f. (Inv.-Nr. 53. 18).

${ }^{14}$ D. L. Carroll, Looms and Textiles of the Copts. First Millennium Egyptian Textiles in the Carl Austin Rietz Collection of the California Academy of Sciences, 168, Abb. 60. 


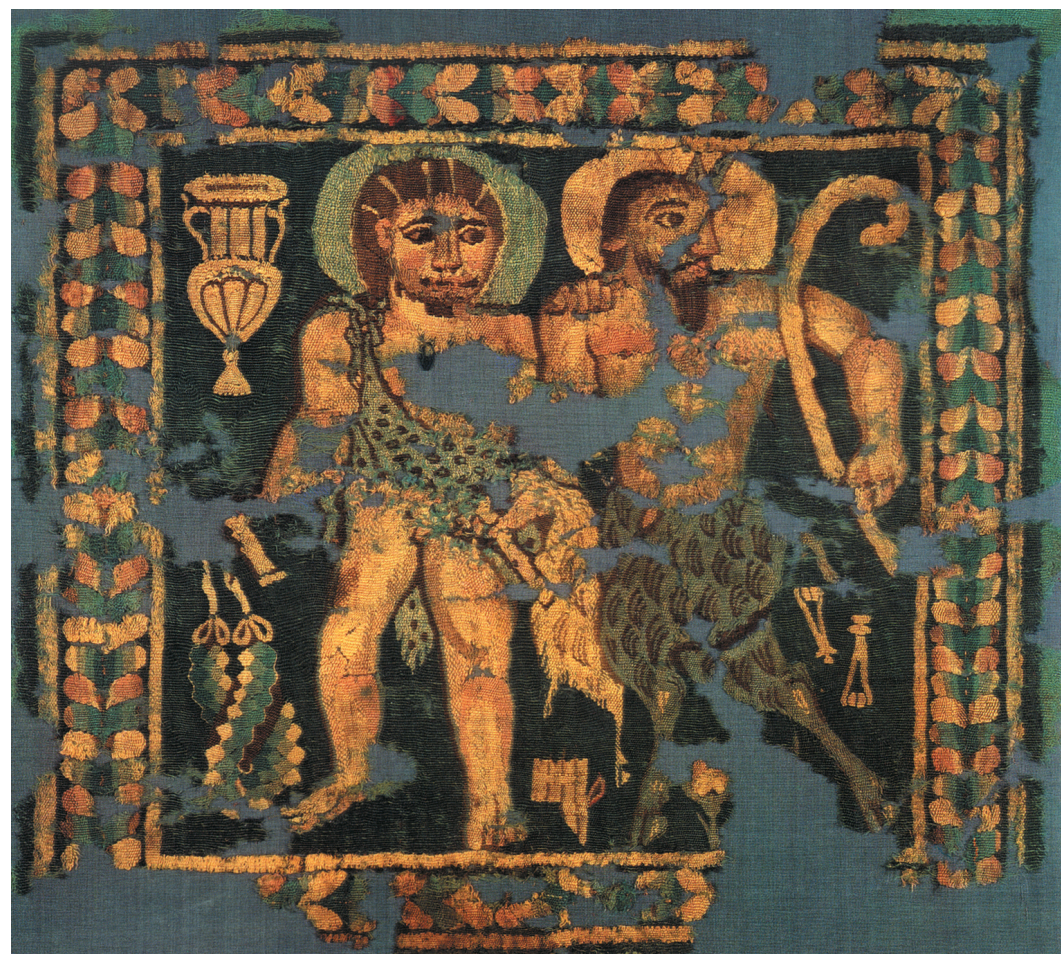

Abb. 6: Quadratisches Besatzstück, Pan und Dionysos, $40 \times 36 \mathrm{~cm}$, Boston, Museum of Fine Arts, Inv. Nr. 53.18 ( Foto nach M.-H. RutschowsCAYA, Coptic Fabrics [wie Anm. 13], 19)
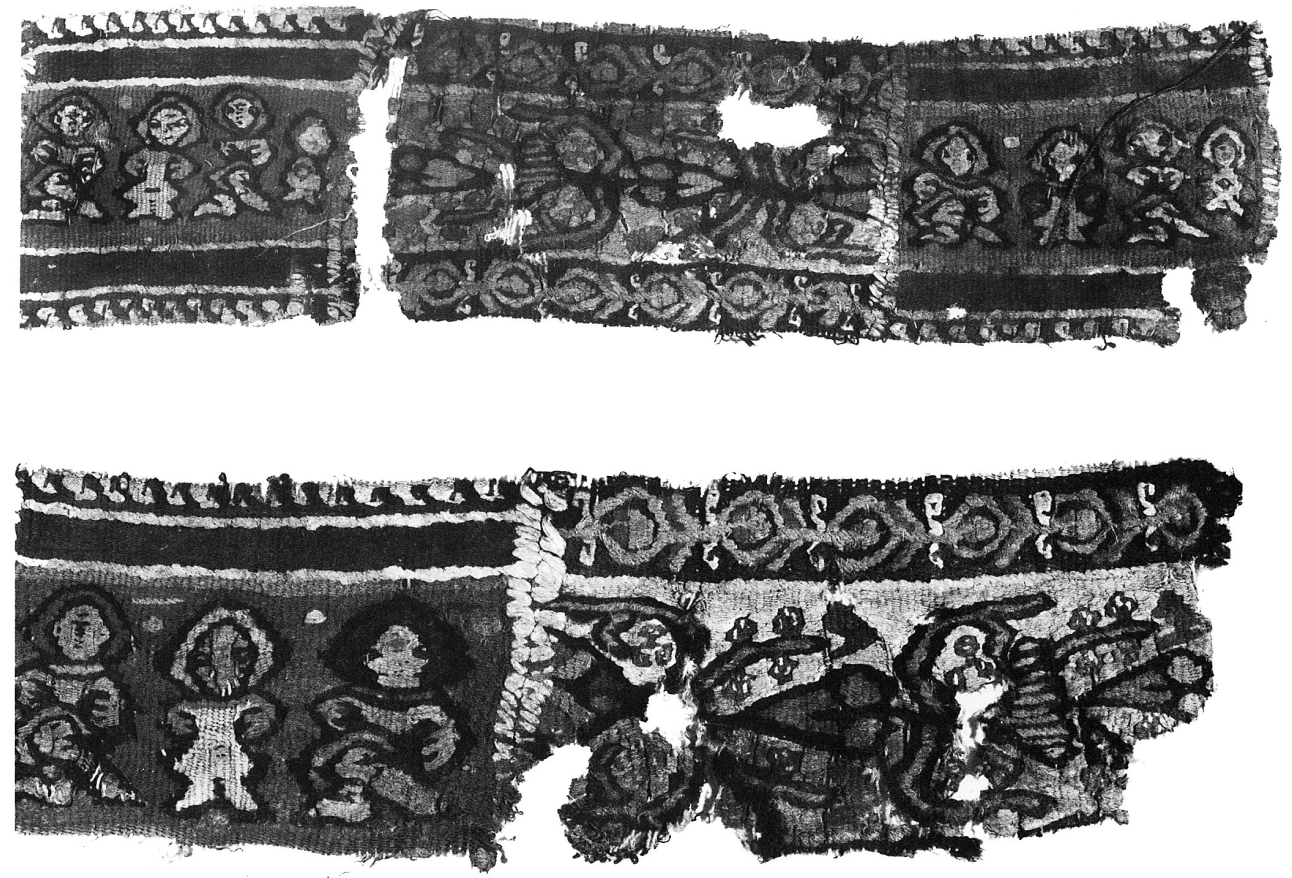

Abb. 7: Zwei Clavusfragmente, $6 \times 18 \mathrm{~cm}$ und $7 \times 26 \mathrm{~cm}$, C. A. Rietz Sammlung (Foto nach D. L. CArroll, Looms and Textiles of the Copts [wie Anm. 8], 168, Abb. 60) 
vollführen dieselbe Bewegung: mit dem einen Bein einen Tanzschritt. Es sind vielleicht nackte Jünglinge (nicht Satyrn oder Silene). Sie tragen einen Tanz- oder Kampfschild, der in vielen Fällen als Tamburin gedeutet werden kann, was ihrer Ikonographie wohl auch entspricht ${ }^{15}$. Diese Deutung als Tänzer im dionysischen Gefolge des bocksbeinigen Pan passt hervorragend in die Thematik des Stoffes.

Zum Abschluss wird das zentrale Medaillon mit den zwei hockenden Figuren und dem stabartigen Gebilde in ihrer Mitte näher zu betrachten sein. Auf einem viereckigen Einsatz ${ }^{16}$ (Abb. 8) aus der Kanegafuchi Sammlung ist dieselbe Darstellungsweise mit zwei Figuren, die den Kopf frontal und den Oberkörper im Profil abgebildet haben, dazwischen das trennende Gebilde, zu sehen. Die Gestalten sind detailreicher ausgeführt und im Unterschied zum Textil T 34 bekleidet. Das stabartige Gebilde, die Armhaltung und der Aufbau mit den vegetabilen Eckornamenten weisen jedoch eine große Ähnlichkeit auf.

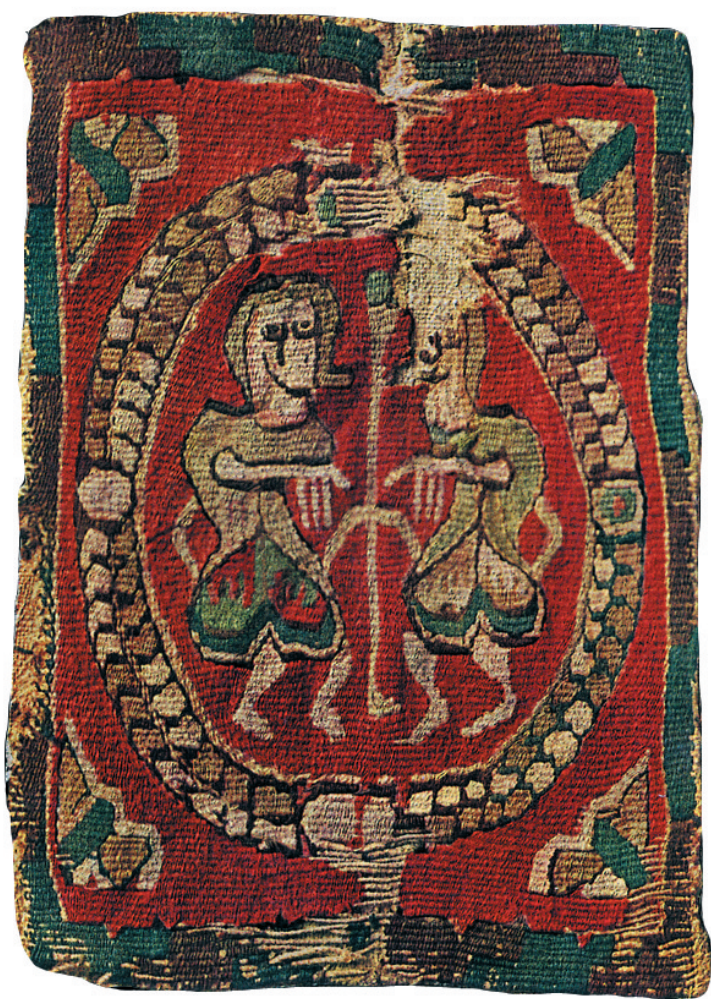

Abb. 8: Viereckiger Einsatz, Kanegafuchi Sammlung (Foto nach K. AKashi [Hg.], Coptic Textiles [wie Anm. 12], Abb. 78)

Diese Darstellung als eine Szene mit Adam und Eva zu interpretieren ist verlockend. Die Figuren des Medaillons der Ärmelborte T 34 scheinen nackt zu sein, bedecken jedoch weder ihre Scham noch ist das Gebilde in ihrer Mitte als Paradiesesbaum oder Schlange zu erkennen. Eines der wichtigsten Zeichen fehlt vollständig, nämlich der Unterschied Mann und Frau. Aus den eben angeführten Gründen enthält das Medaillon auf T 34 wohl keine Adam- und Evadarstellung. Es sind wahrscheinlich vielmehr zwei tanzende Putti zu sehen, die, mit ihren Krotalen schlagend, zum Gefolge des Dionysos gehören. In ihrer Mitte steht ein Lebensbaum oder eine Weinranke, die beide mit dem Dionysoskult eng verbunden $\operatorname{sind}^{17}$.

Heidnische Motive aus der klassischen Antike zusammen mit abstrakten geometrischen und verschlungenen Mustern waren die sich am längsten haltenden Möglichkeiten der Dekoration auf den sog. koptischen Stoffen ${ }^{18}$. Die bekannten mythologischen Motive wurden oft abgebildet, da sie vertraut und verbreitet waren, und man sie daher auch in einem frühchristlichen Kontext verwenden konnte. Die Ärmelborte T 34 transportiert also Mythologisches und Paganes, Pan und sein Gefolge mit Putti und Lebensbaum.

${ }^{15}$ I. Peter, Textilien aus Ägypten im Rietberg Museum Zürich [Sonderausstellung 20. Mai - 14. November 1976]. Zürich 1976, $23 \mathrm{f}$.

${ }^{16}$ K. AKashi (Hg.), Coptic Textiles Vol. 1-16 from burying grounds in Egypt. Kanegafuchi Spinning Company Collection, Abb. 78. Der Autor gibt keine Erklärungen und Erläuterungen zum abgebildeten Stück ab, was es schwierig macht, diese Stoffe zum Vergleich heranzuziehen. Ich möchte das aber trotzdem tun, da einiges wohl vergleichbar ist, und die Textilien daher als Beispiele doch geeignet scheinen.

${ }^{17}$ Siehe Ch. Pflegerl, Eine koptische Ärmelborte der Sammlung Tamerit (wie Anm. 1), 64.

${ }^{18}$ H. Granger-Taylor, The Decoration of Coptic Textiles. In: A. De Moor (Hg.), Koptisch Textiel uit Vlaamse privé-verzamelingen. Coptic Textiles from Flemish private collections [Katalog zur Ausstellung Zottegem 20. November 1993 - 30. Jänner 1994]. Zottegem 1993, 16. 
Die koptischen Weber entwickelten unter Beachtung und Beeinflussung der griechischen, römischen und ägyptischen Kunst und später der islamischen, ihren eigenen Stil. Daraus entsprang etwas Neues, welches kombiniert mit dem Zeitgeschmack der Kopten die sogenannte koptische Kunst ergab ${ }^{19}$. T 34 in die Zeit zwischen das 7.-9. Jh. ${ }^{20}$ zu setzen, scheint ein überlegenswerter Ansatz zu sein ${ }^{21}$.

Mein Dank richtet sich an alle diejenigen, die mich unterstützt und mir bei meiner Arbeit geholfen haben. So möchte ich Herrn HR Prof. Dr. Hermann HARRAuer, Direktor der Papyrussammlung der ÖNB, der es mir ermöglichte, in der Sammlung an „meinem“ Stoff zu arbeiten, und Univ.-Prof. Dr. Renate PILlinger, die mir mit Rat und Tat zur Seite stand, danken. Vor allem aber war es Dr. Ulrike HORAK†, die mein Interesse weckte und die nie müde wurde, meine Fragen zu beantworten.

\section{ABKÜRZUNGEN}

AA $\quad \ldots \ldots \ldots$ Acta Archaeologica

\section{Summary}

A late antique fragment of a Tunic (T 34) from the Tamerit Collection

This late antique textile with the inventory number T 34 is part of a German private collection called "Tamerit". It is currently located at the "Papyrussammlung der Österreichischen Nationalbibliothek". This so called "coptic" piece consists of four rectangular panels, which surround a central medallion. It was placed on the sleeve of a Tunic. The panels probably show the frontal "Pan", accompanied by three dancers. The two "Putti" beside a vegetable creation in the medallion in the center, perhaps a tree of life, fit very well to the panels. Therefore the subject of this textile is a wellknown mythological. The textile T 34 has a good weaving technique and presents the typical "coptic" style.

${ }^{19} \mathrm{Zu}$ Betrachtungen des Begriffes „koptisch“ siehe Ch. Pflegerl, Eine koptische Ärmelborte der Sammlung Tamerit, 82-86.

${ }^{20}$ Die Datierung der sog. „,koptischen Stoffe“ ist grundsätzlich schwierig. Zum einen wurden sie meistens durch Grabräuber oder Sammler aus ihrem Fundzusammenhang gerissen, zum anderen lassen sich keine zufriedenstellenden Chronologien der Stoffe finden, in die man die Ärmelborte T 34 einordnen könnte. Die Technik wurde ebenfalls zu wenig ausführlich bearbeitet, als dass man sagen könnte, diese oder jene Webtechnik ist typisch für eine bestimmte Zeit. Was man versuchen kann, ist, den Stoff anhand von Vergleichsbeispielen einem bestimmten Zeitraum und die besonderen formalen Merkmale des Textils auf Grund von Analysen einer Zeit zuzuordnen (siehe Ch. PfLEGERL, Eine koptische Ärmelborte der Sammlung Tamerit, 86ff.).

${ }^{21}$ In Weiterführung dieser Thematik bearbeite ich die Textilsammlung der Höheren Bundeslehranstalt für Mode und Bekleidungstechnik in 1140 Wien, Herbststraße 104, in einer Dissertation. Die Sammlung umfasst zirka 100 Stofffragmente, die Teil der Graf'schen Sammlung waren. 